\section{LA CRÍTICA LITERARIA DE ALONE Y SU RELACIÓN CON LA PROBLEMÁTICA DE GÉNERO. UNA MIRADA DE ALONE A LA OBRA DE GABRIELA MISTRAL Y MARTA BRUNET}

LITERARY CRITICISM OF ALONE AND ITS RELATIONSHIP WITH THE GENDER QUESTION. A VIEW OF ALONE IN THE WORK OF GABRIELA MISTRAL AND MARTA BRUNET

\section{Resumen:}

\section{Abstract:}

El presente trabajo busca mostrar la relación The aim of this work is to show the relationship que tienen las críticas literarias de Hernan of the literary criticism of Hernan Díaz Arrieta Díaz Arrieta (Alone), ejercidas en medios (Alone), in the press as El Mercurio, La Nación de prensa como El Mercurio, La Nación and Revista Zig-Zag, with the feminine issue, y Revista Zig-Zag, con la problemática from a gender perspective. In order to carry femenina, abordado desde una perspectiva out this work, we use an analysis of content de género. Para llevar a cabo este trabajo se (which is free of criticism) that Alone made aplica un análisis de contenido libre a las about the books of Marta Brunet and Gabriela críticas literarias que Alone realizó a las obras Mistral.

de Marta Brunet y Gabriela Mistral.

\section{Palabras claves:}

KEY WORD: Mistral, Marta Brunet, gender, literary press criticism.

genero, critica literaria periodistica.
La crítica literaria es un espacio de interpretación de obras literarias, ya sea a través de revistas académicas o medios de prensa, lo que la convierte en un importante texto de propagación cultural. De ahí el interés de estudiarla como instrumento social y como comunicación pública cuando se realiza en medios masivos. Los trabajos que apuntan en este sentido no son pocos, entre ellos destacan los trabajos sobre crítica literaria y derechos humanos, realizados por Hernán Vidal, un intento fructífero de indagar en la relación que se produce entre fenómeno textual y social, entendiéndolos como complementarios y mutuamente influyente. Esta investigación indaga la relación de los textos críticos de Hernán Díaz Arrieta (Alone), quién desarrolló una prolífica obra en medios de prensa nacional como "El Mercurio" y "La Nación", con respecto a la situación de marginación de la mujer de los espacios culturales y por qué no públicos. Para esto, la investigación hace uso de una perspectiva de género, como forma de entender las prácticas sociales en las que se inscribe la literatura y por extensión la crítica literaria, como institución permeable a los valores sociales, y que contribuye a que la mujer se encuentre relegada a un papel doméstico.

0.1.- Problema de INVESTIGACIÓN

Es de sabida importancia la obra crítica de Hernán Díaz Arrieta (Alone) por haber hecho de conocimiento público a importantes escritores o al menos haber contribuido a validar sus textos. En este sentido, el presente trabajo ahonda en la crítica literaria que Alone realizó a dos escritoras de vital importancia en la literatura chilena, Gabriela Mistral y Marta Brunet. Ambas ganadoras del premio nacional de literatura y la primera merecedora del Premio Nóbel.

El análisis de las críticas de Alone, realizadas en los diarios El Mercurio y La Nación y en Revista Zig-Zag, a las obras más importantes de estas dos escritoras, tiene como objetivo precisar la relación de sus textos con la situación de la mujer, entendiéndola desde una perspectiva de género, una categoría social que permite explicar la carencia de espacios otorgados a las mujeres. Determinar esto, no sólo señalará la importancia de Alone en la crítica y literatura chilena, cosa ampliamente estudiada, sino en un nivel más amplio, la importancia de la crítica literaria periodística en la transmisión de fenómenos que cruzan la sociedad de manera transversal.

\section{1.- LA PERSPECTIVA DE GÉNERO: ALGUNOS ALCANCES NECESARIOS}

Los estudios que abordan la perspectiva de género se han sucedido con posterioridad a la década del 60 de manera vertiginosa con el fin de integrar una serie de trabajos que 
buscan indagar en las diferencias entre lo masculino y lo femenino, teniendo en cuenta que las mujeres están en una situación, por lo menos, poco favorable.

Para abordar el problema de género, se debe tener presente en primer término que la palabra género no es exclusiva de los estudios sobre la mujer; de hecho hay una serie de divisiones de género en distintas disciplinas. Espinosa y Kautz hacen notar que: "Cuando se habla de género suele pensarse que se trata de una categoría aplicable específicamente a las mujeres, incluso se llega a suponer que género es sinónimo de mujer, incluso en el movimiento feminista, cayéndose en un grave error" (2002: 38) Pearson, Turner y Todd-Mancillas en su libro "Comunicación y Género", nos señalan que hay que comprender una primera distinción sustantiva entre la noción de género y la de sexo. Mientras la segunda obedece a una condición biológica con la que toda persona nace, en la cual un cromosoma Y marca la diferencia en la generación de los caracteres sexuales, la categoría de género es un constructo social, un conjunto de prácticas que son propias de cada cultura y otorgan las características a mujeres y hombres (1993: 27)

La doctora Marcela Lagarde ha realizado estudios sobre género y sus trabajos apuntan en idéntica dirección al señalar que esta categoría es una construcción y realidad cultural, sociológica y psicológica (citada por Espinosa y Kautz, 2002: 41). Esta perspectiva nos abre por tanto la mirada más allá del dato biológico al incorporar a la sociedad como responsable de lo que conocemos como mujeres y hombres.

1.1.- GÉNERO COMO CONSTRUCCIÓN SOCIAL

La categoría género, en una primera aproximación, nace en contraposición a la idea de sexo, pero en específico como lo hace ver Montecinos y Obach tiene un carácter sociocultural, un espectro muy amplio de instituciones que ayudan en la socialización y formación de la identidad (1999). Así por ejemplo, la familia como núcleo básico de la sociedad juega un papel de suma importancia en el traspaso de los valores. Muchas prácticas, como la violencia intrafamiliar o el confinamiento de las mujeres a lo doméstico son traspasadas de padres a hijos, reproduciendo idénticas situaciones (Vargas, 1999). La construcción de esta identidad no es, como lo he dicho, en absoluto de nacimiento sino más bien: El conjunto de disposiciones por el que una sociedad transforma la sexualidad biológica en productos de la actividad humanas y en que se satisfacen esas necesidades humanas transformadas" (Rubin; 1986) El género nos permite, de este modo, abarcar los espacios donde se desarrolla el ser humano, entender la serie de relaciones sociales en que las mujeres han sido confinadas a lo doméstico, a lo privado, siendo desplazada de los papeles actanciales de la sociedad. Se cumplen de este modo las palabras de Shilarowbothan que señala que las mujeres han sido "escondidas de la historia" (citada por Veneros, 2001)
Resita 1825 acional de Culturas y Literaturas, abril 2006

\subsection{1.- Mujer y naturaleza}

La mujer ha sido confinada a lo doméstico y privado, una vida restringida al hogar y los hijos. Henrietta Moore en su libro "Antropología y Feminismo" nos plantea que la subordinación femenina ha sido se asocian a la naturaleza, que es el elemento a vencer sobre todo en la cultura occidental (1996).

"De esta manera, se identifica a los hombres con la sociedad y el interés público, mientras las mujeres siguen asociadas a la familia y, por tanto, a consideraciones particulares o socialmente fragmentadas" (Moore, 1996: 29)

Se producen una serie de pares, siguiendo a la autora, donde queda de manifiesto la oposición entre lo masculino

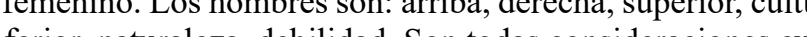

La idea de "mujer objeto" se entiende en este reduccionismo de la mujer a su cuerpo. Entorno al dato biológico se forman una serie de estereotipos y roles que le son asignados a cada mujer por nuestra cultura, contribuyendo las propias mujeres a través de los medios de comunicación a ser reducidas a lo puramente físico y desplazadas de lo cultural.

\subsection{2.- Roles y estereotipos}

Hemos indagado en el concepto de género como ha sido abordado en las ciencias sociales, comprendiendo como hombres y mujeres no son sólo el fruto de la biología sino de las relaciones sociales y pautas culturales que rigen la convivencia social.

"La mujer verdaderamente femenina debe comportarse del modo que se considere socialmente apropiado para su género" (Nicolson, 1997: 34) Los comportamientos de ambos sexos se restringen, de esta manera, a la forma de entender la masculinidad y feminidad dentro de una sociedad particular. Cuando se escapa de estos moldes o estereotipos, las sanciones sociales son inmediatas. En el caso de las mujeres que poseen un carácter fuerte son vistas con recelo y tienden a ser masculinizadas, por el contrario, un hombre dependiente y sumiso es visto como "afeminado".

Es muy difícil, por las sanciones sociales que ello implica, escapar del estereotipo Debido a esto las propias mujeres aceptan en muchas ocasiones los roles asignados. Si se echa una mirada al estereotipo femenino se comprueba que una mujer debe ser ingenua, insegura y preocupada de su hogar. Aún en este tiempo, con copiosa información sobre la situación femenina, se continúa verificando que las mujeres con hijos son menos contratadas que las que no los tienen y a su vez, las mujeres en general menos que los hombres. Esto se debe a que en el estereotipo femenino se incluye su dependencia del hombre. Nacidas de una costilla, deben apoyar y respetar al marido, él por su parte debe ser independiente y triunfador. 


\section{2.- CRÍTICA LITERARIA: PROBLEMATIZACIÓN EN NUESTRO ESTUDIO}

\section{1.- Crítica literaria periodística: alguna de sus características}

Eduardo Guerrero señala que la crítica literaria se ejerce en tres grandes áreas: periodismo, universidad y instituciones formales (1995).Para Manuel Jofré, la CLP se manifiesta en bibliografías, reseñas, notas, comentarios y reportajes sobre libros. Una de sus particularidades es estar escrita para consumo masivo, lo que implica menores niveles de conocimiento del tema teórico que la crítica académica, que se dirige a gente más especializada y se vale de los avances en teoría literaria, lingüística y social. Para entender de manera clara las características de la CLP hay que tener presente tres grandes diferencias que la hacen particular.

-Lenguaje: se trata de un discurso caracterizado por ser claro y sintético, esto hace que en muchas ocasiones se le acuse de falta de originalidad.

-Espacio: en relación a esta característica hay consenso en señalar que los espacios destinados a ejercerla van en franca decaída en los medios locales, esto coincide con el llamado "apagón cultural" (Guerrero: 90).

En relación a este llamado "apagón cultural" cabe recordar el intento llevado a cabo por el desaparecido diario El Metropolitano; que en sus comienzos publicaba un semanal llamado -Diagonal- dedicado exclusivamente a temas literarios y culturales. Al poco andar, dicho suplemento, debió cambiar de formato a una revista que sólo trataba de literatura en sus últimas páginas y el resto era farándula. Finalmente desapareció no sólo la revista sino también el diario, lo que evidencia un proceso mucho mayor de concentración mediática y disminución de los espacios de todo lo que huela a crítica, producto neto de un mirada postmodernista.

Asistimos a un fenómeno de desplazamiento de lo cultural a espacios íntimos e ínfimos, norma general en los medios de masas. Esto reduce notoriamente los artículos y notas a simples reseñas bibliográficas empobreciendo su contenido. Aun así, en los espacios donde puede seguir haciéndose es: "un ejercicio de síntesis interesante y que debe continuar" (Guerrero 1995: 91) La CLP, en palabras de Rodrigo Cánovas, tiene como función la información y valoración de las obras literarias, estableciéndose un contacto directo con el lector masivo de los periódicos. El problema que presencia Cánovas en la CLP es la subestimación del lector, al que se le considera "insulso, arratonado, talquino" (1995: 115). Esto lleva a que los textos sean simplones, que no vayan más allá de señalar cuál libro comprar y cuál no. Un empobrecimiento del contenido es lo que Cánovas observa como resultado.
ISSN: 1885-3625

En razón de esta problematización que tiene la CLP, tanto desde el punto de vista ético como estético, se hace interesante revisar la relación que ha tenido la crítica literaria con su contexto cultural.

\section{2.- ¿La CLP como género periodístico?}

Es preciso agregar una contextualización problemática a lo que se ha señalado sobre CLP, para incluirla en un contexto (movedizo, como veremos) que permita enfrentar estos discursos en el marco de estudios mediáticos. Hay que tener presente que dichos discursos al ser expuestos en medios de prensa, a diferencia de la crítica literaria académica, pueden serclasificados en los denominados “génerosperiodísticos". Veamos algunas clasificaciones para luego intentar problematizarlas y por qué no, apostillarlas. Casasús y Ladavèze en su libro "Estilo y géneros periodísticos" (1991) nos resumen de manera didáctica una clasificación en 4 grandes grupos, según distintos autores:

-Informativos: denominados así por Albertos, Núñez y Gomis y van Dijk. Llamados narrativos en Borrat y Aullón.

-Interpretativos: en Albertos y Fagoaga: Evaluativos en Ladevèze y van Dijk; Descriptivos en Aullón y Borrat.

-Argumentativos: Aullón, Borrat, Ladevèze; Evaluativos en van Dijk.

-Instrumentales o prácticos en van Dijk.

Esta separación obedece en primer término a una división clásica entre opiniones y hechos, Comment/Story. Es así como Van Dijk citado por Casasús distingue entre esquemas narrativos (relatos) y esquemas argumentativos (los artículos), admitiendo la existencia de un tercero llamado "práctico" que corresponde a los textos de cambio de divisas, obituarios y otros. Más articulada es la proposición de Borrat, quién en 1981 propuso la división entre: narrativos, descriptivos y argumentativos. Lo más interesante del trabajo de este autor es la identificación en los textos de los denominados "topoi" latinos ó las " $W$ " en inglés. De este modo Borrat articula una división más retórica de los textos. Según Borrat, en los textos narrativos predominan el qué, quién, cuándo; en los descriptivos el qué, quién, dónde y en los argumentativos el por qué y cómo (Casasús y Ladevèze, 1991) Borrat luego complejiza estos 3 géneros agregando los narrativos simples donde predominan el qué, quién y cuándo; el narrativo explicativo donde prevalecen el qué, quién, cuando, por qué y cómo. En los descriptivos simples está presente mayoritariamente el qué, quién y el dónde y los descriptivos explicativos: qué, quién, dónde, por qué y cómo.

Los argumentativos sufren la misma división: argumentativos simples; por qué y cómo; argumentativos explicativos donde predominan: por qué, cómo, dóndey cuándo. 
LaCLPseinscribeenlatipologíadeBorratenunargumentativosimplepuespredominala presentación de la obra literaria, donde el crítico expone las razones por las que considera quesusjuiciosestéticossonacertadosycómosearticulalaobraparaproducirsignificación. Género Explicativo Simple o en palabras de Rivadeneira un Género de Opinión es visto como un complejo sistémico de interpretaciones, valoraciones semánticas de propósitos pragmáticos que se materializan en el producto que aparece en medios, periódicos, revistas y radios. La clasificación de la crítica en un género argumentativo, según la tipología de Borrat, es útil para acercarnos a un análisis textual mediático, compartimos con Casasús que Borrat olvida aspectos en los que ha trabajado Van Dijk sobre contexto y cognición del discurso, por un lado, y elementos éticos y políticos en los que se involucran los productores de los discursos.

Las palabras de Rivadeneira, que incluyen a la crítica en un apartado sobre el género de opinión son igualmente válidas, pues Casasús indica citando a Albertos, que “Cuando el periodista utiliza la exposición o la argumentación se sitúa en el mundo de la opinión y su mensaje periodístico recibe el nombre de comentario" (1991: 91). Se entiende la idea de comentario en el sentido que Borrat entrega de un texto que responde al por qué y cómo de manera fundamental, con un carácter subjetivo y libre pero en ningún caso hay que considerarlo antojadizo y simple, de hecho debemos tener presente que el género de opinión está sujeto a reglas de juego limpio, que Jofré ha denominado "estrategia ética" u honestidad intelectual y responsabilidad (1997) algo muy difícil de establecer en un mundo muy mercantilizado.

\subsection{1.- Apostillas a los géneros periodísticos. Prolegómenos para una discusión futura}

Como puede verse, esta es una clasificación bastante clásica de los géneros periodísticos. Es posible pensar que esta división busca mantener la vieja separación entre hechos y comentarios y por tanto, la objetividad periodística. Sin embargo, es necesario dejar al menos esbozadas algunas dudas que los mismos autores de este artículo tienen.

Las investigaciones más recientes ven como la división en géneros estáticos es un poco ruda y violenta. La hibridez como llama Rodríguez (2004) estaría poniendo en cuestión al menos la forma tradicional de concebir lo periodístico. Un ejemplo de esto lo encontramos en los relatos periodísticos (García Marquez, Truman Capote, etc) transformados en libros. ¿Literatura o periodismo?. Pareciera que ambos, ni lo uno ni lo otro, sino un tercero complejo y más rico.

Estoes patente en el caso de la CLP pues muchas veces nosencontramos con relatos que narran las peripecias deun sujeto (en sus viajes, por ejemplo) y su relación con los libros de sugustoodisgusto.Estopuedeconcebirsecomoundesplieguedecomentariossobrelibros,
Revista Internacional de Culturas y Literaturas, abril 2006

pero también como la historia (ficcional o real) de un sujeto particular (novela o cuento) Pareciera que los géneros son bastante promiscuos y es necesario, a la luz del presente, darle un grado de inestabilidad a la propia (re) contrucción que hemos planteado en 3.1 y que más allá de entregar certezas nos obliga a repensar los lindes entre lo comentado/informado, real/ficcional, periodismo/literatura.

\section{3.- COMPOSICION DEL CUERPO DE ANÁLISIS}

El corpus de estudio está compuesto de críticas realizadas por Alone a las obras de Gabriela Mistral y Marta Brunet en los diarios El Mercurio y La Nación y en Revista Zig-Zag, los artículos fueron recogidos del libro "Alone y los premios Nacionales de literatura" compilado por Pedro Zegers y un trabajo de biblioteca del autor de esta investigación en la Biblioteca Nacional, sección "Referencia y Críticas". Se trata de una obra dispersa y difícil de conseguir dado el mal estado en que se encuentran los textos. La delimitación para la búsqueda es de dos tipos:

1.- Textos producidos en la primera mitad del siglo pasado, hasta el año 65 (19001965) Esto se debe a que las obras más importantes de las autoras se produjeron entre estos años.

2.- La temática de los textos trata sobre las obras de las autoras o hechos relevante en relación con su trabajo, premios, hechos importantes sobre su obra y en último término la muerte de la autoras, como homenaje.

Debido a lo antiguo de la obra de Alone se incluyen algunos textos de la revista Zig-Zag bajo los mismos parámetros anteriores, una revista de gran tiraje $y$ masividad, que no nos aleja de nuestros objetivos. Los textos de Alone, sobre todos los publicados en El Mercurio en la sección "Crónica Literaria" son de gran extensión, muchos superan las tres o cuatro carillas por lo que el estudio de unas pocas críticas es suficientes, creemos, para un análisis adecuado y extenso.

\section{1.- La selección del crítico y otras precisiones metodológicas pertinentes}

La selección del crítico se debe en primer término a su relevancia en la crítica chilena. Determinar en el marco de este trabajo si esta posición es merecida o no, escapa de los alcances de la investigación y es algo de suma dificultad por lo que implica establecer la veracidad de un juicio de valor con el que muchos pueden o no estar de acuerdo.

El hecho de tomar los trabajos de este crítico se debe no sólo a su importancia, fuera de discusión, sino más bien a la tribuna a la que tuvo acceso y que lo hace un autor interesante. Por otro lado, y a modo de crítica, creo que los estudios a su obra han tratado más bien sobre la biografía, en ningún caso menospreciada, pero que no 
permite inferir de su obra datos relevantes. Se habla mucho de su relación con autoras y autores, como Shade, Iris, Mistral, Neruda y otros, pero poco se aborda su crítica en un estudio que permita descomponerla, como objeto de análisis.

Resumiendo: se trata en el caso de la elección del crítico de una elección por el público al que accedía, pues el cuerpo de análisis considera trabajos realizados en medios de prensa de alcance masivo Como guía para el análisis textual, expongo la siguiente hipótesis guía: En las criticas de Alone se puede precisar y presenciar una relación con la situación de la mujer en la sociedad y la literatura

Para indagar en esta hipotesis realizó un análisis descriptivo de las relaciones entre los textos y la situación de la mujer, basado en un análisis de contenido, pues este procura comprender los datos, "no como un conjunto de acontecimientos físicos, sino como fenómenos simbólicos, y abordar su análisis directo “ (Krippendorf, 1990: 7).

\section{4.- ANÁLISIS DEL CORPUS}

\section{1.- Sobre Marta Brunet:}

En el texto titulado Marta Brunet y La moral literaria1 la presencia de la mujer y una problemática más amplia desde el género está dada en primer término por el tema, donde una lectora escribe a Alone para contar que los libros de Brunet leídos en silencio son agradables, pero en voz alta causan "escrúpulo". Alone, ante la pregunta de la lectora, elabora una respuesta en la que va caracterizando la prosa de Brunet como sólida y desenvuelta. A su juicio habla rectamente y sin rodeos, "sin perderse por nieblas sentimentales", lo que causa pavor, sobre todo cuando es escrita o pronunciada por mujeres, pues es algo impensado para una mujer de "bien".

Cabe recordar lo que se consignó en el marco teórico cuando planteaba que la literatura escrita por mujeres es considerada muchas veces como palabras pasajeras, que se pierden en sentimentalismo, este estereotipo hace que la literatura de mujeres no sea estudiada con seriedad. Por otro lado hay que considerar que este texto es de 1927, donde las aprensiones para que una mujer ejerciera la literatura eran fuertes. Las mujeres se reducían a la esfera doméstica y no podía soportarse que alzaran la voz. Las alusiones textuales ayudan al crítico a evidenciar lo claro que habla Brunet de las cosas difíciles, lo que la hace una mujer de excepción, pero agrega: "esto no es para niñitas de monjas, ni aún para caballeros que hablen de moral con letras mayúsculas". En un claro cuestionamiento a los hombres y mujeres que no permiten a una mujer expresarse con la misma libertad que los hombres, un derecho natural y fuera de todo cuestionamiento.

\section{ISSN: $1885-3625$}

Alone aclara su punto de vista "Sería necesario destruir la idea de la literatura femenina tradicional, hecha de dulces de almíbar(...). Sería necesario inculcarles a los lectores la convicción de que un autor no es hombre ni mujer, ni soltero ni casado, ni de buena o mala compañía, sino que es una inteligencia, un corazón, una voz de humanidad dotada de la facultad de transmitirse." Por medio de una pregunta casi al cierre, Alone señala abiertamente la situación de la mujer en esos años "¿Cómo no asombrase de que aparezca, de pronto, una escritora? Hace poco se luchaba porqué las mujeres pudieran escribir con su nombre, sin esconderse". El enmascaramiento de las mujeres que utilizaban seudónimos masculinos, es otro de los elementos que nos destaca como la mujer ha sido desplazada y lo sigue siendo, menos cuantitativa que cualitativamente de lo cultural.

En Marta Brunet, Premio Nacional de Literatura 1961 la relación con la cuestión de género está dada por la insistencia en caracterizar a Brunet como una mujer de carácter fuerte, que marca una diferencia con el prototipo de mujer convencional: "Nada de sentimentalismos, ningún retorcimiento ni ambigüedad seudofilosófica, tan de moda en este tiempo dentro del elemento femenino tocado de intelectual" Al igual que Gabriela Mistral, Alone no podría considerarse un feminista sino un progresista que ve en la situación de la mujer una problemática cultural seria en nuestro país. El no estar adscrito a grupo alguno, autoriza a Alone para criticar al propio movimiento feminista, tal como lo hace la Mistral que ve en estos grupos errores fatales, al intentar igualarse en todo a los hombres. Se puede ver, que incluso esta visión es aún más actual y vigente que los feminismos radicales. Se trata de entender a un sujeto particular, que no tiene ni debe, igualarse con los hombres en el afán de lograr reivindicaciones.

Este texto concluye señalando lo justo del recibimiento y lo justo de presentarla como modelo a los jóvenes. Los merecimientos de esta autora están dados por el juicio a su obra y no por el hecho de ser mujer, lo que de igual modo constituiría una discriminación de género, por el hecho de marcar una diferencia bueno/malo entre hombre y mujer. Bienvenida a Marta Brunet es un relato que insiste en la presentación de los rasgos de personalidad que considera distintivos en la autora. Quienes la criticaron por su manera de decir las cosas, sin tapujos, deben sentirse más mal que la afectada, pues sus obras tienen un merecido espacio en la literatura nacional e incluso universal. El texto Marta Brunet mediante alusiones textuales sucesivas, escogidas con pinzas de un relato de la autora, muestra la escena de un velatorio que permite a Alone reflexionar sobre la vida Brunet y el penoso momento contingente de su muerte. La utilización de una retórica rica:

"Es su voz, una de sus voces" 
Su relación con la problemática femenina se manifiesta de manera franca al destacar que su temperamento nuevo para la época la llevó a estar en dos polos: la admiración y el rechazo, lo que le causaba innumerables inconvenientes.

En el texto de 1943 Aguas Abajo Alone hace un uso más intensivo de las alusiones textuales con párrafos enteros de citas, que buscan explicitar que dichos textos son una prosa "difícil de encontrar en escritores latinoamericanos", con lo que apoya la visión presentada en las crónicas anteriores. La presentación de la autora y su obra como un objeto lingüístico y estético complejo hace que Alone rompa con lo que hemos llamado en el marco teórico, la invisibilización de la mujer en la historia, literaria en este caso, al asumir que su obra es digna de ser estudiada, como lo indica en el texto, incluso en "tesis universitarias" debido a su complejidad. En Don Florisondo por medio de alusiones textuales se inserta el diálogo de una mujer que tiene una filosofía de vida que se aleja del molde de mujer sometida a su marido, tan propio de la primera mitad del siglo pasado. De hecho es el hombre quien está a merced de esta señora, que cuenta cuatro maridos, siendo conviviente del último. “Con el primero me casé por todo lo que hay que casarse y ¡viera cómo me salió el codenado!... me abandonaba, se iba al pueblo a remoler, se curaba, me trataba peor que un perro. Hasta que al cabo se murió." "Voy a decir a su merced el secreto para hacer feliz a los hombres, para tenerlos seguros, hay que agarrarlos por el miedo a encontrarse cualquier día sin mujer"

Se trata de una buena filosofía indica Alone, una filosofía con mayúsculas, en la que hay que consignar la situación de marginalidad que vivían las mujeres. La cita no es descuidada ni fortuita, está escogida para mostrar la situación de dominación hombre/mujer, pero donde esta vez "la señora" sale airosa por medio de estratagemas legitimadas por la necesidad.

Como señalé, el resto de los textos sigue una presentación más apegada a las obras de Brunet. Lo interesante es notar como Alone rescata que una mujer se levante con personalidad distinta. Hay que considerar, por otro lado, el entorno mediático donde se desenvolvía Alone, un medio como El Mercurio, en la mayoría de los textos, que siempre se ha identificado con una corriente conservadora y de derecha política, lo que podría haber restringido la aparición de críticas donde se muestra a una mujer diferente, que escapa del molde conservador.
Revista Internacional de Culturas y Literaturas, abril 2006

4.2.- Gabriela Mistral

Hay que consignar que al igual que en el caso de Marta Brunet los ojos de Alone se vuelven sobre Gabriela cuando aún no ha publicado sus primeros libros y mucho menos tiene la relevancia de décadas más tarde. En este caso se trata de textos extensos, como en el caso de Desolación, que tiene 7 carillas y por tanto su sólo análisis bastaría para un estudio profundo. El texto se inicia haciendo alusión a lo difícil que resulta para las mujeres dedicarse a la literatura y cuando lo hacen se trata de mujeres de clase alta que tienen como mecenas a intelectuales de talla que las protegen de las furibundas críticas del medio social: “Extraño caso no sólo en nuestra tierra, sino en la historia universal, el de esta mujer que no nació en cuna extraordinaria y, sin embargo, ante de publicarse su primer libro, tiene por todos los países de su lengua mayor gloria que muchos grandes autores clásicos."

Como en el caso de Brunet, Alone trabaja fuertemente en la exposición de las características de su personalidad fuerte y áspera, que la hacen distinta y por tanto "problemática". Luego introduce una pregunta que inicia el debate: ¿Gabriela es la primera poetiza o el primer poeta? El problema de género es patente al preguntarnos si es mejor que sea la primera poeta mujer o un poeta, entendiendo esto en lo masculino que implica la palabra, ¿acaso uno es más importante que el otro?. Lo que queda claro es que existe una diferencia, que se percibe como negativa, pero que Alone da a entender como innecesaria dada la relevancia que tiene Mistral.

Podría pensarse que para Alone el poeta es una especie de Between en el que no puede concebirse el género y por eso señala que no tiene sentido la pregunta. La visión que predomina en Alone es concebir al poeta como constructor de nuevos espacios. Muy al modo de Baudelaire, el poeta es ese ser en que coexisten las contradicciones. El texto continúa con las citas textuales que corresponden a la obra analizada, vista como versos plagados de pasión y fuego. Al igual que Brunet, su capacidad para hablar sin tapujos ni rodeos de sus pasiones coloca a la sociedad en la disyuntiva de aceptarlas por su franqueza o rechazarlas por la osadía impertinente. Tala es muy similar en composición a Desolación y se insiste en mostrar esa personalidad fuerte que saca sus efusiones para gritarlas al mundo. A pesar del apego que tiene Mistral a la religión, "la profunda contextura moral de su poesía", que podrían haberla hecho titubear a la hora de expresar el amor pasional, logra elevarse por sobre las moralinas y funda una moral propia. Reminiscencias de Gabriela Mistral señala que luego de tener una infancia dolorosa y de luchar por ingresar a la escuela pedagógica para ser maestra, "Estaba admitida. Pero cuando llegó, con su pequeño equipaje, le dijeron que el Consejo, en su último acuerdo, había rechazado su solicitud. La causa la supo mucho más tarde: unos versos 
publicados por ella en un periódico local y que fueron considerados "panteístas"..." Un ejemplo más de cómo Alone logra insertar tangencialmente la problemática que aquejaba a Mistral y Brunet por dedicarse a la literatura. No se trata de que la mujer escriba poco, no hay estadísticas al respecto, pues muchas lo hacen en secreto aún ahora y eso impide un cálculo preciso, pero lo hacen con mucha frecuencia, resistiendo en ocasiones las críticas más descarnadas. En Con Gabriela Mistral, fragmentos de un diario de viaje se logra ubicar con mayor nitidez, la dificultad que tienen las mujeres para salir del anonimato, para no ser "esposas de..." sino autónomas. Citando a una escritora, no nombrada, nos señala que para las mujeres la gloria no será nunca sino el duelo resplandeciente de la felicidad.

Mistral calzaba con esto a la perfección: al estar profundamente marcada por su infancia y adolescencia, vivía una tristeza en la que Alone descubre su fuerza poética y que arrancaba de su falta de atractivo físico. El resto de los textos del corpus, como es el caso de Gabriela Mistral están construidos sobre la base de alusiones textuales, donde Alone pone énfasis en develar los misterios de las poesías mistralianas. Esto al igual que en el caso de Brunet es relevante pues Alone analiza de manera sistemática, sin sesgo de género, y antes de ser "famosa", la obra de una mujer. Recados contando a Chile cae en el mismo caso al ser la crítica literaria directa de una obra en prosa de la autora. De la misma manera ocurre con el resto de los textos.

En los trabajos de Alone puede establecerse una concientización de la problemática de la mujer no sólo en la literatura sino en la sociedad en general. Esto desde una óptica de género obedece a una cuestión sociocultural de desplazamiento de la mujer de papeles actanciales como la literatura. Este problema obedece a una situación que Alone ve arraigada en nuestra sociedad cuando, por ejemplo, la lectora siente vergüenza al escuchar las palabras claras de Brunet, y en el intelectual cuando alza su voz diciendo que la literatura femenina es sinónimo de sentimentalismo. Como lo observa agudamente Alone, las mujeres como Brunet y Mistral que no cumplen con los requisitos estereotipados de mujer débil, dependiente y apegada al hogar, son miradas negativamente excluyéndolas. Es necesario entender la discriminación de género como un problema transversal, donde la literatura no es la excepción.

\section{5.- A MODO DE CONCLUSIONES}

1) Al finalizar este trabajo es menester volver sobre el comienzo, cuando planteábamos que nuestros objetivos eran verificar la importancia de la crítica literaria en la transmisión de fenómenos sociales más amplios y que cruzan a la sociedad transversalmente. En este sentido, vemos que la crítica literaria es un espacio fructífero para investigar la
Revista Internacional de Culturas y Literaturas, abril 2006

problemática femenina, un tema que en las últimas décadas ha entrado con fuerza en la Ciencias Sociales.

2) Se puede constatar por medio del estudio sistemático de sus críticas que claramente en los textos de Alone hay una relación con el medio circundante con respecto a la situación femenina. Esta relación se establece, en ocasiones, de manera abierta y franca y en otras de manera implícita, por el tema tratado o simplemente por hacer visibles al público como objetos estéticos complejos, la obras de estas dos escritoras chilenas.

3) Los textos de Alone entregan lineamientos para entender el momento cultural que se vivía en materia de relación de género. Se concluye, de esto último, la necesidad de tomar a la crítica literaria ejercida en medios de prensa, en el justo espacio que corresponde. Esto es, como medios de resistencia o mantenimiento de problemas mayores como pueden ser las violaciones de los derechos humanos o la marginación de la mujer.

Debido a esta defensa, si bien no panfletaria y llamativa como algunos esperarían, Alone ha sido llamado feminista. Creo, como ya dije, que el caso de Alone es muy similar al de Mistral, se trata más bien de un pensamiento progresista, sensible al tema, no de un feminismo propiamente tal. De este modo la hipótesis que nos planteamos al principio, que era la de mayor abstracción se encuentra superada y nos permite aventurar que: La crítica literaria periodística es un discurso social relevante en la transmisión de valores y en la resistencia o perpetuación de estereotipos.

REFERENCIAS BIBLIOGRÁFICAS

Alone, Los cuatro grandes de la literatura chilena del siglo XX, Chile, Zig.Zag, 1963.

----, El vicio impune, Santiago, RIL, 1997.

Barbieri, T., Sobre la categoría género, una introducción teórica-metodológica, Ediciones de las mujeres, № 7, 1992 .

Casasus,J.;Ladevèze, L., Estiloy géneros periodísticos, Barcelona, Ariel comunicaciones, 1991.

Carrasco, I., "La crítica literaria en tiempos de crisis", en Alonso, M.; Rodriguez, M; Triviños, G (Eds.), La crítica literaria chilena, Concepción: Anibal Pinto, pp. 35-42, 1995

Espinoza, L.; Kautz, J., Análisis de los discursos públicos realizados por políticos en Chile, pre y post $I V$ conferencia mundial de la mujer, Tesis conducente al grado de licenciado en comunicación social. Universidad de La Frontera, 2002.

Eltit, D.; Brito, E., Poderes, Imaginarios y Textualidades http://www.utem.cl/trilogia/ Volumen 16 n 25 26/p 16 5.html. 
Galaz, C., "La contribución del periodismo al desarrollo", en Actas VI seminario interdisciplinario de estudio de género en las universidades chilena, Chile, Dolmen, pp 337-347, 2002.

Guerra, L., La mujer fragmentada, Santiago, Cuarto Propio, 1995.

Grau, O., Ver desde la mujer, Santiago, Cuarto Propio, 1990.

Huidobro, C., "Alone y las escritoras chilenas", Revista de libros , pp.6-8. 2001.

Jofré, M., Teoría Literaria y Semiótica, Santiago, Universitaria, 1997.

Los diarios de Alone, La Segunda, 26/07/2000.

Mistral. G., Pensamiento feminista -mujer y oficios -, Chile, RIL, 1998.

Moore, H., Antropología y Feminismo, 1996.

Montecinos, S.; Obach, Género y Epistemologías: Mujeres y Disciplina, Santiago, Cuarto Propio, 1999.

Nieves, M; Rodríguez, M.; Triviño, G., La Crítica Literaria Chilena, Concepción, Anibal Pinto, 1997.

Nicolson, P., Poder, género y organizaciones, Nircea, 1997.

Pearson; Turner Y Todd Mancillas, Género y medios de comunicación, 1993.

Pellegrini, S (1978). “La mujer y los medios de comunicación de masas”, en Covarrubias,

P; Franco, R. (Eds.) Chile, mujer y sociedad, Chile, Fondo de las Naciones Unidas para

la infancia, pp.583-590, 1978. Krippendorff, K., Metodología de análisis de contenido,

Barcelona, Paidós, 1997.

Van Dijk, T., Racismo y análisis crítico del discurso, Barcelona, Paidós, 1997.

----, La ciencia del texto, Barcelona, Paidós, 1992

Veneros, D., "Historia de las Mujeres y Relaciones de Género en Chile", Revista de Ciencias Sociales de la Universidad José Santos Ossa, III , 7-26. 2001.
Revista Internacional de Culturas y Literaturas, abril 2006

-Bienvenida a Marta Brunet; El Mercurio, 16 de Noviembre de 1961, pp. 3

-Marta Brunet; El Mercurio, Crónica Literaria; 30 de octubre de 1967, pp. 19

-Don Florisondo, El Mercurio, (sin fecha por deterioro del periódico)

2.- Gabriela Mistral

-Desolación; La Nación; 3 de junio de 1923, pp. 4

-Tala; La Nación; 29 de mayo de 1938, pp. 2

-Gabriela Mistral; El Mercurio; 20 de enero de 1957, pp.7.

-Reminiscencias de Gabriela Mistral; Zig-Zag N²122; 22 de noviembre de 1945, pp. 27-28.

$-3^{\circ}$ Edición de la poesías de Gabriela Mistral; La Nación; 22 de Mayo de 1926

-Recados contando a Chile, por Gabriela Mistral;:El Mercurio, Crónica Literaria; 3 de noviembre de 1954

-Con Gabriela MistraL, fragmentos de un diario de viaje; Zig-Zag N²703; 12 de mayo de 1957. -Gabriela Mistral en México; El Mercurio, Crónica Literaria; (sin fecha por deterioro)

Anexo de críticas por autora

1.- Marta Brunet

- Marta Brunet y la moral literaria; La Nación; 31 de julio de 1927, p.7

-Marta Brunet; Aguas Abajo; El Mercurio, Crónica Literaria; 28 de noviembre de 1943.

-Montaña Adentro: El Mercurio; Crónica Literaria;19 de septiembre de 1954

-María Nadie; El Mercurio; Crónica Literaria; 17 de noviembre de 1957

-Montaña Adentro: la querella contra el criollismo; Zig-Zag; 10 de Julio de 1954

-Marta Brunet, premio Nacional de literatura 1961; El Mercurio; Crónica Literaria; 9 de septiembre de 1961, pp. 5 